
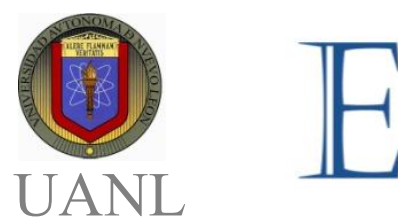

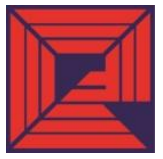

Facultad de Economía

\title{
Determinantes del aumento o disminución de empresas: Análisis por entidad federativa y estrato de edad
}

\section{The Determinants of the Increase and Decrease in the Number of Firms: An Analysis by State and Age Stratum}

\author{
Rolando I. Valdez* \\ Eder J. Noda Ramírez
}

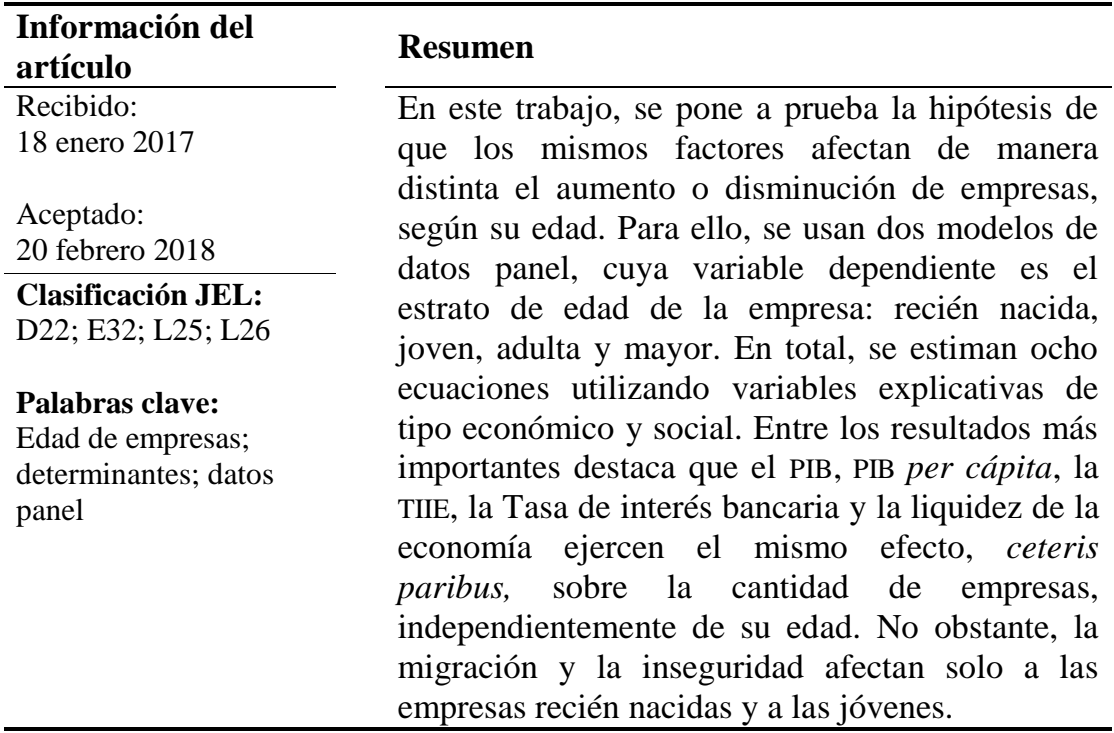

\footnotetext{
"Universidad Autónoma de Baja California. Av. De las Limas 9342-11A, El Refugio. C.P. 22253. Tijuana, Baja California. rvaldez35@uabc.edu.mx

${ }^{* *}$ Universidad Iberoamericana. San Luis Potosí No. 13 dpto. 202, col. Belisario Domínguez, C.P. 91055, Xalapa, Veracruz. eder.noda@gmail.com
} 


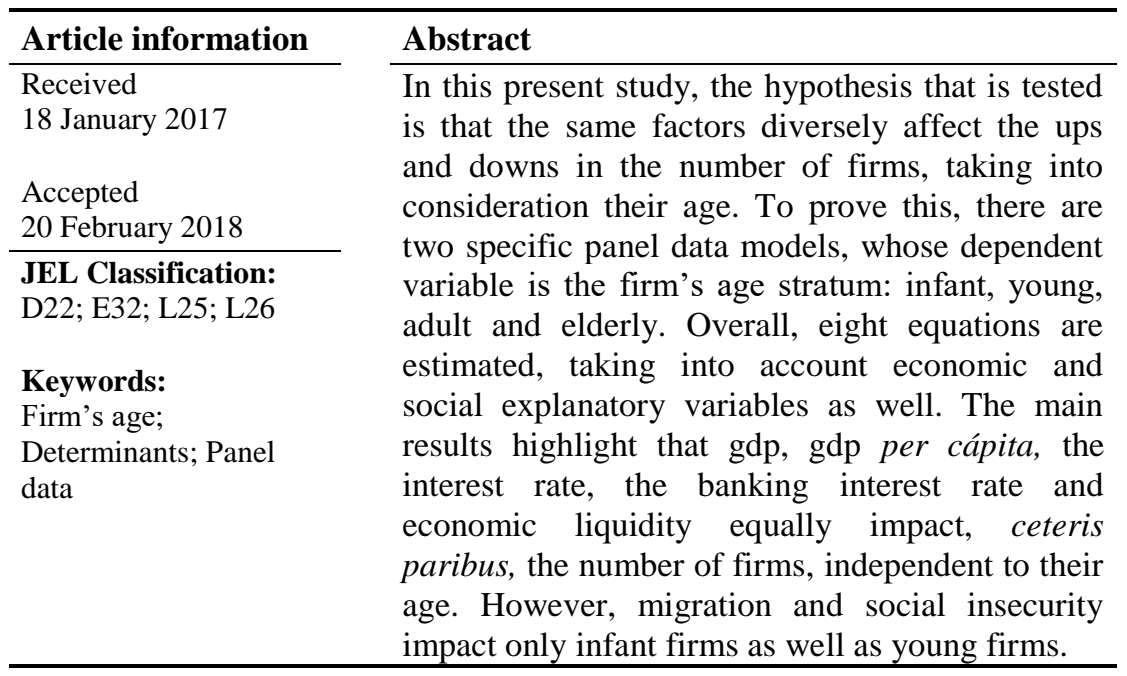

\section{Introducción}

El estudio de la edad de las empresas es por sí mismo escaso en la literatura económica. Sin embargo, algunos textos abordan este tema a partir del análisis microeconómico; específicamente desde la teoría del productor, que tiene que ver con la escala óptima, la cual está relacionada, a su vez, con los costos. Por otro lado, otros trabajos empíricos están enfocados en explicar la entrada y salida de empresas, es decir, aquellas que nacen y las que culminan un ciclo de vida; lo cual en cierta forma limita el alcance de dichos trabajos, al solo estudiar los dos extremos de la edad de las empresas. Más aún, la relación entre agregados económicos con la edad de las empresas también está limitada al estudio del nacimiento de estas, así como a su salida del mercado. En la literatura, se ha omitido el análisis para la edad de las empresas como tal, cuestión que este documento aborda, ya que aquí se contrasta la hipótesis de que los mismos factores provocan, en diferente dirección, el aumento (o la disminución) de la cantidad de empresas según su edad. Lo cual es importante conocer para comprender qué variables de la economía estimulan que existan más o menos empresas de alguna determinada edad. El presente trabajo se diferencia de otros, porque se enfoca en cuatro estratos de edad, y utiliza las mismas variables explicativas para corroborar la hipótesis previamente descrita.

Una característica común e inherente a las empresas es el ciclo de vida. A diferencia de los seres vivos, en las firmas es indefinido, ya que la permanencia de estas en el mercado no depende de causas biológicas, sin embargo, pasan por diferentes etapas desde que nacen, maduran y llegan a una edad mayor; algunas se mantienen funcionando, y otras dejan de hacerlo 
en cualquiera de las etapas de su ciclo. Esta característica permite diferenciar a las empresas entre sí, ya que afrontan los aspectos internos y externos de múltiples maneras.

El ciclo de vida está asociado con la edad de las empresas. La primera etapa del ciclo, el nacimiento, es en donde inicia actividades la firma. Si bien existe un periodo previo de planeación que también es importante, por el tema del financiamiento, los procesos legales, fiscales, etc., no está considerado en el ciclo de vida del negocio, debido a que es un aspecto que afecta más al emprendedor que a la firma. Después del nacimiento, vienen diversas etapas, que pueden caracterizarse de diferentes maneras, según el enfoque de cada investigador. No obstante, en las etapas posteriores al nacimiento de la empresa, se define el rumbo de la misma; es aquí donde se toman decisiones respecto de mantenerse o salir del mercado, considerando alguna experiencia y retroalimentación sobre el funcionamiento de la firma.

Lo anterior deja claro que, a cada edad, las empresas enfrentan diferentes escenarios que implican la consideración de diversos factores, tanto internos como externos, que les permitan continuar, crecer, expandirse, mejorar, entre otros. Esta diferenciación implica que para comprender fenómenos de entrada y salida de empresas, debe distinguirse la edad en la que estas se encuentran, así como el contexto socioeconómico. Es poco informativo enmarcar en el mismo análisis a todas las empresas que inician o terminan operaciones, si no son reconocidas por sus edades; sobre todo para las segundas, ya que la salida puede ocurrir en cualquier momento. Por lo tanto, interesa saber las causas para algunas de las etapas por las que cruza cada empresa.

Dentro de las causas que implican una mayor o menor existencia de empresas de determinada edad, están los agregados económicos; interesa saber en qué sentido y magnitud afectan, los cambios de la economía en su conjunto a los grupos de empresas, según sus edades. Este tipo de información es útil para comprender, desde otra perspectiva, un posible ciclo de vida de las firmas.

Este trabajo tiene como objetivo evaluar los efectos que tienen algunos agregados económicos y sociales en la existencia de empresas para un determinado estrato de edad, identificando a las recién nacidas, jóvenes, adultas y mayores, para las entidades federativas de México en los años 2003, 2008 y 2013. En consecuencia, se espera corroborar la hipótesis de que los mismos factores ejercen impactos diferenciados para cada estrato de edad de las empresas.

\section{Revisión de la literatura}

Uno de los trabajos pioneros que considera el papel de la edad de las empresas es el de Evans (1987), quien establece las relaciones que existen entre el crecimiento, el tamaño y la edad de las mismas. Dentro de los 
hallazgos más importantes de este autor, se encuentra que la tasa de crecimiento de la firma es decreciente con respecto al tamaño y a la edad; lo que implica que las empresas mayores o adultas crecerán a tasas más bajas que las jóvenes o las recién nacidas. También, el autor encuentra que la edad de la firma incrementa las probabilidades de que esta sobreviva. Con estos resultados, está en posición de rechazar la ley de Gibrat, que indica que el crecimiento y el tamaño de las empresas son independientes entre sí, para una industria determinada en un periodo dado. Y, por transitividad, la edad que se encuentra asociada a un mayor tamaño, también aplicaría para dicha ley.

Más aún, el autor encuentra que la edad de la firma es un determinante importante de su crecimiento, la variabilidad de este crecimiento y también de su disolución. Por lo tanto, su estudio abarca una dinámica amplia sobre el ciclo de vida de las empresas, que es muy importante estudiar, ya que es necesario comprender los momentos en donde se tienen más probabilidades de sobrevivir o, contrariamente, de disolverse. Otros trabajos, que también encuentran evidencia de que la edad de la empresa tiene una relación negativa con el crecimiento de la misma, son los de Mansfield (1962) y Dunne y Hughes (1994), por mencionar algunos.

El hecho de que el crecimiento de las empresas sea decreciente conforme su tamaño aumenta, se debe a que dejan de observarse aquellas firmas de tamaño pequeño que crecen lentamente (Mansfield, 1962). Sin embargo, cabe el razonamiento del mismo autor cuando ejemplifica que, de acuerdo con la ley de Gibrat, una empresa que vende 100 millones de dólares al año tiene la misma probabilidad de duplicarse que una que vende 100 mil dólares anuales. Lo anterior implica que, la lógica detrás de la ley de Gibrat es que los ingresos (o costos) de las firmas son proporcionales a su tamaño, lo cual es cuestionable actualmente; por ejemplo, a la hora de considerar el sector de servicios basados en conocimiento.

La edad de la empresa tiene una relación inherente tanto con el crecimiento como con la supervivencia. Una empresa adulta o mayor, sin duda ha sobrevivido durante un periodo de tiempo y debió haber crecido, en un sentido amplio; aunque, no necesariamente, pues mantener un tamaño constante también puede considerarse como sobrevivencia: un negocio exitoso no necesariamente tiene que expandirse o incrementar su tamaño, sino permanecer en el mercado.

Las empresas deben enfrentar situaciones internas y externas en el espacio donde se desempeñan, con el único objetivo de permanecer en el mercado. Algunas firmas logran sobrevivir, crecer o expandirse, mientras otras no logran dicho objetivo y terminan saliendo.

Las fluctuaciones macroeconómicas evidentemente afectarán de forma distinta a cada empresa, según las características que esta tenga. La edad es una característica relevante que es poco evaluada en los trabajos relacionados 
con la supervivencia de las empresas. Un trabajo pionero en este rubro es el de Boeri y Bellmann (1995), quienes evalúan los efectos de los agregados económicos en el crecimiento y la supervivencia de firmas. Los autores encuentran que las empresas son insensibles a las fluctuaciones económicas, sin embargo, cuando desagregan las firmas por cohorte de edad, muestran una ligera sensibilidad ante dichas fluctuaciones. La explicación que los autores ofrecen está relacionada con el tamaño que alcanzan las empresas mayores, mismas que ante los shocks externos, tienen la posibilidad de encogerse, mientras que para las firmas recién nacidas o jóvenes, asociadas a un tamaño pequeño, su primera opción es la salida.

Sin embargo, no solo el tamaño de la empresa es determinante en su salida, las empresas más jóvenes tienen problemas con el capital humano porque carecen de empleados con altos conocimientos en el campo financiero. Existen diversos factores que permiten que las firmas sobrevivan, por ejemplo, empresarios con más educación y con mayor financiamiento desarrollan negocios que permanecen más tiempo en el mercado (Bates, 1990). Una mayor educación se refleja en mejores capacidades gerenciales, y el financiamiento inicial se traduce en empezar la empresa con la máxima capacidad posible. Si bien el mayor financiamiento incrementa las posibilidades de éxito de la firma que comienza, aquellos emprendedores con mayores recursos financieros tienen mayores probabilidades de que sus negocios prosperen (Holtz-Eakin, Joulfaian, \& Rosen, 1994).

Por su parte, las empresas más viejas son incapaces de adaptarse a los cambios externos que se generan en la economía (Tornhill \& Amit, 2003). Estos cambios pueden estar relacionados con el mercado, o incluso con ciertos aspectos institucionales del país según donde residen.

Las restricciones de liquidez condicionan el nacimiento de empresas, no obstante, no se ha explorado cómo afecta este factor a las empresas jóvenes o adultas, por lo tanto, el acceso al crédito es un factor determinante para el éxito de los negocios. García-Quevedo et al. (2014), evalúan la hipótesis de que las restricciones financieras y de liquidez, por parte de la demanda, afectan más a las empresas jóvenes que a las adultas o mayores, en España, durante el periodo 1990 a 2008. Estos autores encuentran evidencia consistente con dicha hipótesis, es decir, la condición del mercado orienta en buena medida a la permanencia de las firmas jóvenes; por tal motivo, es necesario evaluar cómo afectan el acceso al crédito y la liquidez en el nacimiento de empresas. Por otro lado, también es interesante conocer el efecto sobre las firmas que ya han sobrevivido por algún tiempo. $\mathrm{Al}$ respecto, Sánchez-Vidal y Martín-Ugedo (2008) prueban la hipótesis de que, según la edad de la firma, recurren a diferentes tipos de financiamiento, particularmente, las empresas adultas y mayores recurren a préstamos formales, tales como bancos y otras instituciones crediticias, mientras que las recién nacidas, lo hacen con fondos propios. Su evidencia les impide sostener 
esta afirmación, debido a que obtienen resultados opuestos a la hipótesis nula: las firmas adultas y mayores se financian con recursos propios gracias a la obtención de beneficios económicos, mientras las empresas recién nacidas y jóvenes adquieren considerables deudas para financiarse. Estos resultados son consistentes con los de Pfaffeymayr, Stöckl y Winner (2013), quienes encuentran que las empresas mayores tienen menores índices de deuda que las empresas jóvenes o las recién nacidas, resultado también consistente con el de Huynh y Petrunia (2010).

El bajo costo del capital se asocia a un tema de liquidez y financiamiento al que tienen acceso los emprendedores, por lo tanto, en el nivel agregado, la estabilidad de precios y la tasa de interés son fundamentales para el acceso al desarrollo de negocios, independientemente de su edad; sin embargo, la magnitud con que cada variable afecta a un determinado grupo de empresas jóvenes o adultas, es desconocida.

Un resultado que explícitamente no destaca, pero sin duda es una muestra de que el entorno macroeconómico importa, y por lo tanto debe ser considerado, fue expuesto por Pfeiffer y Reize (2000), sobre las empresas iniciadas por desempleados tanto en Alemania del Este como del Oeste, mostrando mejores condiciones y probabilidades de supervivencia las de la segunda región que las de la primera. Aunque los autores no destacan estas relevantes diferencias macroeconómicas, conviene hacer esta observación acerca de la Alemania dividida en dos regímenes de diferente organización de la actividad económica, una orientada por el mercado (la del oeste), y la otra por el Estado (la del este).

Bhattacharjee et al. (2009), sostienen que el ambiente macroeconómico es el más importante para el desarrollo de los negocios; mientras la quiebra de empresas se extiende principalmente cuando existen periodos de recesión económica, la compra de estas crece mientras la economía está recuperándose. En contraste, Highfield y Smiley (1987) hacen una afirmación en el mismo sentido, sobre todo porque enfatizan los determinantes macroeconómicos para la entrada de empresas, por ejemplo, las bajas tasas del crecimiento del PIB e inflación o el alto desempleo. Audretsch y Acs (1994) hacen el mismo hallazgo sobre el desempleo, como principal determinante del nacimiento de empresas. Cabe señalar que es desconocida la relación entre el desempleo y las empresas adultas y mayores, por lo tanto, es necesario analizar este tema.

Por otro lado, Ilmakunnas y Topi (1999) elaboran un trabajo empírico para medir las influencias microeconómicas y macroeconómicas en la entrada y salida de firmas en la industria manufacturera de Finlandia. Sobre las segundas, sus resultados no son concluyentes, sin embargo, mencionan rubros interesantes que la literatura aborda, como el desempleo; también consideran la oferta de crédito y las restricciones por el lado de la tasa de 
interés real, esta última es causa fundamental de la salida de empresas en el Reino Unido; aunque el crédito y los beneficios también influyen en que las firmas fallen (Liu, 2004).

En otro estudio, Benavente y Külzer (2008) investigan las causas de la entrada y salida de las empresas. Su estudio demuestra que el PIB y el desempleo tienen un impacto negativo, es decir, propician más la salida que la entrada de firmas.

En México, es escaso el estudio de los efectos macroeconómicos sobre las empresas según su edad. Se pueden identificar casos que analizan por separado algunos de estos agregados y, principalmente, se enfocan en el emprendimiento. Por ejemplo, Hernández-Trillo, Pagan, y Paxton (2005) estudian el capital inicial, las microempresas y la eficiencia técnica. Estos autores investigan las condiciones de eficiencia iniciales de las microempresas, a través de un modelo de fronteras estocásticas, y obtienen como resultados que las empresas que reciben préstamos bancarios de prestamistas o, en general, financiamientos formales, presentan una escala más eficiente que las que son inicialmente financiadas por familiares o amigos. Por lo tanto, el tipo de financiamiento es importante considerarlo como un elemento al que pueden responder de forma diferente las empresas.

Rangel y Torre (2015) investigan el impacto que tienen las variables como la desregulación para la entrada de empresas, la fortaleza financiera de los estados, el crimen y la rentabilidad potencial de mercado en los costos para la entrada de empresas a nivel estatal. Estos autores encuentran que los esfuerzos por desregular la entrada, así como la fortaleza financiera de los estados son dos factores que disminuyen el costo de la entrada de firmas al mercado, mientras que el crimen y altos niveles de PIB per cápita lo elevan.

Los trabajos hechos para México concentran su atención principalmente en el emprendimiento, mientras que la literatura que considera empresas jóvenes, mayores o adultas es prácticamente inexistente, en parte debido a que la información para llevar a cabo dicho análisis tampoco existía; sin embargo, algunos datos están disponibles para el uso público a partir de 2014.

En este trabajo se busca considerar también a las firmas con mayor edad además de las nacientes, debido a que "las empresas de edad diferente reaccionan de manera distinta al mismo conjunto de perturbaciones", Boeri y Bellmann (1995, pág. 495); también presentan diferentes ciclos dinámicos, según sean jóvenes o adultas (Fort, Haltiwanger, Jarmin, \& Miranda, 2013).

Dentro de la revisión de la literatura, fueron identificadas diferentes variables que empíricamente han sido utilizadas para evaluar el impacto sobre la entrada y salida de firmas, así como la edad de estas, estas variables son mostradas a continuación en el cuadro 1, también son presentados los trabajos en donde las utilizan. 


\section{Cuadro 1}

Variables que determinan entrada, salida y edad de las empresas Variable Trabajo

PIB Bhattacharjee et al. (2009); Highfield y Smiley (1987); Benavente y Küzler (2008).

PIB per cápita Bhattacharjee et al. (2009); Rangel y Torre (2015).

Desempleo

Highfield y Smiley (1987); Audretsch y Acs (1994); Boeri y Bellmann (1995); Ilmakunnas y Topi (1999);

Pfeiffer y Reize (2000); Benavente y Küzler (2008).

Riesgo

Boeri y Bellmann (1995); Rangel y Torre (2015).

Innovación

Boeri y Bellmann (1995).

Financiamiento

Holtz-Eakin, Joulfaian y Rosen (1994); Sánchez-Vidal y Martín-Ugedo (2008); Pfaffermayr, Stockl y Winner (2013); Huyn y Petrunia (2010); Liu (2004); Hernández-Trillo, et al. (2005); Bates (1990).

Tasa de interés Ilmakunnas y Topi (1999).

Liquidez García-Quevedo, Pellegrino y Vivarelli (2014).

Fuente: Elaboración propia.

\section{Metodología y datos}

Los trabajos revisados en el apartado previo carecen de modelos teóricos a los que pueda recurrirse para justificar teóricamente la inclusión de variables para explicar la existencia de empresas. En otras palabras, la literatura existente sobre la edad de las empresas y los agregados socioeconómicos carece de justificación teórica. Ante la ausencia de un modelo teórico para explicar la edad de las empresas, es necesario recurrir al método inductivo para especificar un modelo econométrico que permita aproximar las variables que deben considerarse para el objetivo propuesto, tomando como referencia las identificadas en la revisión de la literatura.

Por hipótesis, es esperado que los mismos factores afecten de forma diferente la existencia de empresas según su edad, la suma de los factores puede aumentar o disminuir la cantidad de empresas en algún estrato de edad determinado, es decir, 


$$
\begin{gathered}
Y=f\left(\sum X\right) \\
\vdots \\
Z=f\left(\sum X\right)
\end{gathered}
$$

En donde, $Y$ es la cantidad de empresas de una edad determinada y $Z$ es la cantidad de empresas de otra edad, excluyente a las que están en $Y$; mientras que $X$, es una matriz de variables explicativas. La sumatoria de estas explican, en su conjunto, la cantidad de empresas por estrato de edad.

En otras palabras, el valor esperado de empresas en el estrado de edad $Y$ y $Z$ es una función lineal de los siguientes elementos,

$$
\begin{gathered}
E\left(y_{i t} \mid \mathbf{x}_{i t}, c_{i}\right)=\beta_{0}+\mathbf{x}_{i t} \boldsymbol{\beta}+c_{i} \\
\vdots \\
E\left(z_{i t} \mid \mathbf{x}_{i t}, c_{i}\right)=\gamma_{0}+\mathbf{x}_{i t} \boldsymbol{\gamma}+c_{i}
\end{gathered}
$$

En donde $y_{i t} \ldots z_{i t}$ son variables que caracterizan la cantidad de empresas de la edad $y$ a la $z$, en cada unidad $i$ en el periodo $t$. Mientras que $\mathbf{x}_{i t} \boldsymbol{\beta}=$ $\beta_{1} x_{i t 1}+\beta_{2} x_{i t 2} \ldots+\beta_{k} x_{i t k}$ y $\mathbf{x}_{i t} \boldsymbol{\gamma}=\gamma_{1} x_{i t 1}+\gamma_{2} x_{i t 2} \ldots+\gamma_{k} x_{i t k}$. Por su parte, $c_{i}$ es un efecto no observado para cada $i$, que será explicado más adelante.

Con base en lo anterior, el planteamiento general del modelo econométrico es como sigue,

$$
y_{i t}=x_{i t} \beta+c_{i}+d_{t} \theta+u_{i t}
$$

Note que ha sido incluido un elemento $d_{t}$ que varía en el tiempo, pero que es el mismo para las entidades federativas, mismo que debe entenderse como factores que son iguales para todas las observaciones de la muestra, que cambian en el tiempo, pero no entre las $i$. El término $u_{i t}$ es un error idiosincrático que cambia tanto entre las observaciones como en el tiempo y sigue una distribución normal, con media cero y varianza constante.

Con la estructura expuesta en la ecuación 3, es necesario que la especificación econométrica considere una transformación logarítmica debido a que se buscan relaciones de largo plazo entre las variables dependientes y las explicativas, así como efectos del tipo céteris páribus, por lo que la ecuación a estimar es expresada como sigue: 


$$
\begin{aligned}
\ln \left(E M P_{i t}^{k}\right)=c_{i}+ & \ln \left(P I B_{i t}\right) \beta_{1}+\ln \left(P I B p c_{i t}\right) \beta_{2}+\ln \left(D E S O_{i t}\right) \beta_{3} \\
& +\ln \left(R E M_{i t}\right) \beta_{4}+\ln \left(M I G_{i t}\right) \beta_{5}+\ln \left(H O M_{i t}\right) \beta_{6} \\
& +\ln \left(C D E S_{i t}\right) \beta_{7}+\ln \left(C C O M_{i t}\right) \beta_{8}+\text { TIIE }_{t} \beta_{9} \\
& + \text { TIBANC }_{t} \beta_{10}+\ln \left(L I Q_{t}\right) \beta_{11}+u_{i t}
\end{aligned}
$$

En donde se tienen $k=4$ variables dependientes, cada una de estas es la cantidad de empresas en un estrato de edad para la entidad federativa $i$, en el año $t$. Los censos económicos de los años 2004, 2009 y 2014, que a su vez contienen información de 2003, 2008 y 2013, ofrecen esta información por cuatro estratos de edad, mismos que son: 1) recién nacidas, 2) jóvenes, 3) adultas, 4) mayores. Las primeras son empresas con menos de 3 años de antigüedad, las segundas se encuentran en un rango de 3 a 5 años. Por su parte, las adultas son aquellas empresas que tienen más de 5 años hasta 10 , mientras las mayores tienen más de 10 años (INEGI, 2014).

El siguiente cuadro contiene estadísticos descriptivos de los cuatro estratos de edad de las empresas, en donde se indica la cantidad de observaciones, las cuales son 96 debido a que son 32 entidades federativas y tres años en los que son observadas dichas variables. La distribución del promedio de los cuatro estratos es relativamente uniforme por años, no obstante, las empresas adultas y mayores muestran un incremento considerable, debido a que, en el periodo de 10 años, estas prácticamente se duplican, fenómeno que no es observado con las empresas recién nacidas y jóvenes.

Los antecedentes históricos y las dinámicas internas de las entidades federativas conducen en buena medida la actividad económica. Los estados se distinguen en aspectos como su talla demográfica, factores geográficos, entre otros, que naturalmente los llevan a tener diferencias entre sí. Estas disparidades repercuten en la vocación estatal de la actividad y, con esto, en la dinámica de las empresas. Es esperado que algunas entidades tengan mayor capacidad emprendedora que otras, de igual forma, existen algunas que mantienen a las empresas más longevas del país y que, con base en la literatura, generan fenómenos económicos que es necesario estudiar.

Tlaxcala tenía el liderazgo en empresas recién nacidas en el año 2003, tal como es mostrado en la figura 1, ya que poco más de $40 \%$ de sus empresas eran de reciente creación; mientras que en el Distrito Federal, la proporción de empresas recién nacidas era de poco más de $24 \%$, siendo la más baja de todo el país. A Tlaxcala le siguen las entidades de Chiapas, Hidalgo y Quintana Roo con mayor cantidad de empresas recién nacidas. En esta última, junto con Tlaxcala, se localizan la menor proporción de empresas mayores, con aproximadamente $20 \%$ del total. 


\section{Cuadro 2}

Estadísticos descriptivos de las variables dependientes

\begin{tabular}{|c|c|c|c|c|c|c|}
\hline & Variable & Obs. & Media & $\begin{array}{c}\text { Desv. } \\
\text { Estándar }\end{array}$ & Min. & Max. \\
\hline \multirow{4}{*}{ 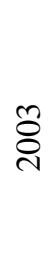 } & $\begin{array}{l}\text { Recién } \\
\text { nacidas }\end{array}$ & 32 & 30073 & 27679 & 4924 & $\begin{array}{c}14119 \\
2\end{array}$ \\
\hline & Jóvenes & 32 & 18723 & 16320 & 3587 & 75007 \\
\hline & Adultas & 32 & 17839 & 15436 & 3428 & $\begin{array}{l}65090 \\
13606\end{array}$ \\
\hline & Mayores & 32 & 27276 & 26749 & 4991 & 0 \\
\hline \multirow{4}{*}{$\stackrel{\infty}{\stackrel{\infty}{ᄋ}}$} & $\begin{array}{l}\text { Recién } \\
\text { nacidas }\end{array}$ & 32 & 36655 & 32905 & 7041 & $\begin{array}{c}16727 \\
9\end{array}$ \\
\hline & Jóvenes & 32 & 23633 & 20577 & 4834 & 99276 \\
\hline & Adultas & 32 & 22037 & 18571 & 4446 & $\begin{array}{l}82470 \\
14577\end{array}$ \\
\hline & Mayores & 32 & 34051 & 30460 & 6483 & 3 \\
\hline \multirow{4}{*}{$\stackrel{m}{\stackrel{n}{d}}$} & $\begin{array}{l}\text { Recién } \\
\text { nacidas }\end{array}$ & 32 & 36953 & 32957 & 8143 & $\begin{array}{c}16746 \\
3\end{array}$ \\
\hline & Jóvenes & 32 & 19504 & 16966 & 4493 & 82250 \\
\hline & Adultas & 32 & 31718 & 28545 & 6227 & $\begin{array}{c}13772 \\
5\end{array}$ \\
\hline & Mayores & 32 & 44035 & 38824 & 8827 & $\begin{array}{c}17534 \\
7\end{array}$ \\
\hline
\end{tabular}

Fuente: Elaboración propia con datos de INEGI, 2003, 2008, 2013. 


\section{Figura 1}

\section{Distribución porcentual de las empresas según edad, 2003}

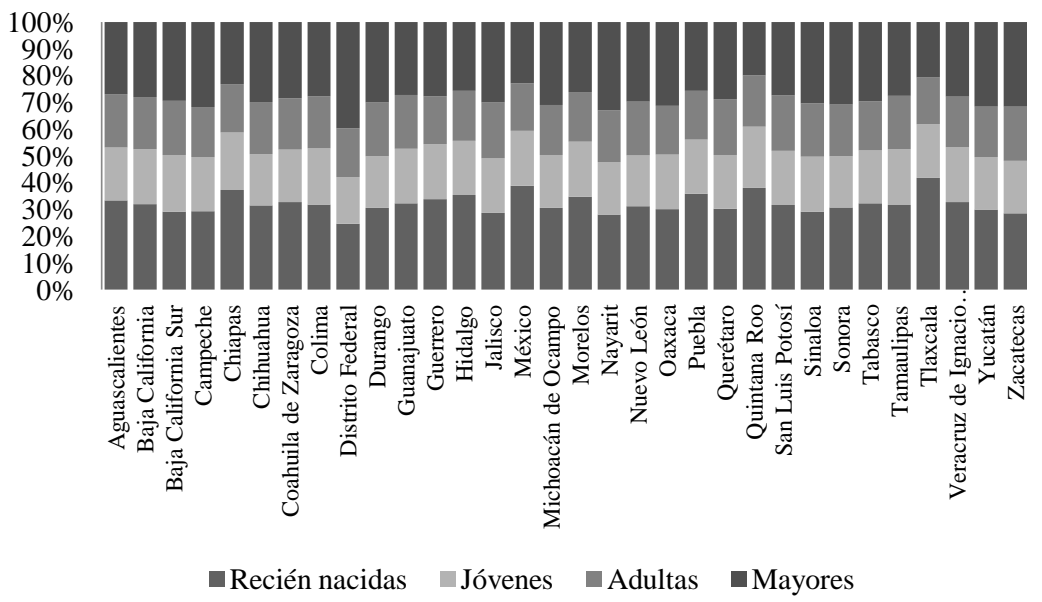

Fuente: Elaboración propia con datos de INEGI, 2003.

El Distrito Federal poseía en 2003 la mayor proporción de empresas mayores, ya que es una entidad en donde históricamente se ha concentrado la actividad económica $\mathrm{y}$, con esto, las empresas han permanecido durante un periodo considerable de tiempo.

Cabe señalar que la proporción de empresas jóvenes y adultas representan alrededor de $20 \%$ en la mayoría de las entidades, los valores para ambos grupos de edad oscilan entre $17 \%$ y $21 \%$, con lo cual las diferencias entre las entidades residen entre las empresas recién nacidas y mayores.

En la figura 2, se muestran las distribuciones porcentuales de las empresas según su edad, para cada entidad federativa, para el año 2013. Los cambios son notables en las empresas jóvenes, adultas y mayores debido a que es en donde se observan los principales movimientos en la distribución porcentual. Para este año, tanto Tlaxcala como Hidalgo son las entidades con mayor proporción de empresas recién nacidas; sin embargo, en este periodo de quince años esta proporción para Tlaxcala se redujo de $41 \%$ a $33 \%$. Las empresas jóvenes incrementaron su dispersión porcentual, pues ahora los valores oscilan entre $12 \%$ y $19 \%$, que pertenecen a Zacatecas y Quintana Roo, respectivamente. La proporción de empresas adultas va desde $21 \%$ hasta $24 \%$ en las entidades federativas, con lo que representan un bloque muy homogéneo para estas. 
Figura 2

Distribución porcentual de las empresas, según edad, 2013

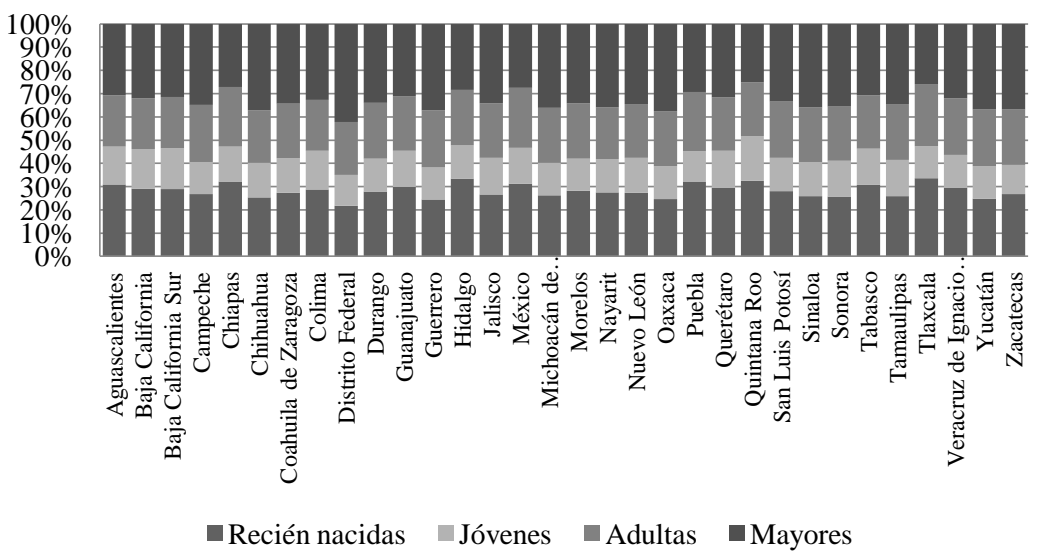

Fuente: Elaboración propia con datos de INEGI, 2013.

La proporción de empresas mayores reporta la mayor variabilidad entre las entidades federativas, debido a que los porcentajes van desde $25 \%$ hasta $42 \%$, que pertenecen a Quintana Roo y el Distrito Federal, respectivamente.

Con lo anterior, es posible señalar la existencia de un ciclo de madurez de las empresas en México, ya que puede observarse cómo varían las proporciones de un censo a otro para cada rango de edad de las firmas, lo cual explica que los estratos de las adultas y las mayores sean cada vez de mayor tamaño.

Por su parte, las variables independientes fueron seleccionadas con base en la revisión de la literatura. La mayoría de ellas refleja algún aspecto económico, tales como el PIB, que representa el ingreso de cada entidad, incluso el tamaño de esta; el PIB per cápita, como un indicador de desarrollo; la cantidad de personas desocupadas, que es utilizada en varios de los trabajos previamente citados; el flujo de créditos otorgados, tanto por la banca de desarrollo como la banca comercial, ambos como fuentes de financiamiento, que es una de las variables en donde más centran su atención los trabajos que estudian este tema; la tasa de interés tanto interbancaria como bancaria debido a que reflejan el costo del dinero, así como los incentivos que puede tener la población para obtener rendimientos de su dinero, ya sea en el mercado de capitales o en el mercado de bienes; la liquidez, medida a través de la masa monetaria, considerando solo el circulante de efectivo, misma que refleja - de alguna manera - la política económica del país. También fueron incluidas variables de tipo social, como las remesas, que también son un tipo de financiamiento no formal: la migración. Las defunciones por homicidios es una variable incluida para controlar el aspecto de la inseguridad en las entidades federativas. 


\section{Cuadro 3}

Descripción de las variables independientes

\begin{tabular}{|c|c|c|c|}
\hline Variable & Descripción & $\begin{array}{l}\text { Unidad de } \\
\text { medida }\end{array}$ & Fuente \\
\hline PIB & $\begin{array}{l}\text { Producto Interno Bruto por entidad } \\
\text { federativa en precios constantes, base } \\
2008 \text {. }\end{array}$ & $\begin{array}{l}\text { Millones de } \\
\text { pesos }\end{array}$ & INEGI \\
\hline PIBpc & $\begin{array}{l}\text { Producto Interno Bruto per cápita por } \\
\text { entidad federativa, precios constantes, } \\
\text { base } 2008 \text {. }\end{array}$ & $\begin{array}{l}\text { Millones de } \\
\text { pesos por } \\
\text { persona }\end{array}$ & INEGI \\
\hline DESO & $\begin{array}{l}\text { Población desocupada de los últimos dos } \\
\text { trimestres del año previo y los dos } \\
\text { primeros trimestres del año en curso por } \\
\text { entidad federativa. }\end{array}$ & $\begin{array}{l}\text { Población } \\
\text { promedio }\end{array}$ & INEGI \\
\hline REM & $\begin{array}{l}\text { Remesas familiares por entidad } \\
\text { federativa. }\end{array}$ & $\begin{array}{l}\text { Millones de } \\
\text { pesos } \\
\text { (reales) }\end{array}$ & Banxico \\
\hline MIG & Migración neta interestatal. & Población & CONAPO \\
\hline HOM & $\begin{array}{l}\text { Defunciones por homicidio por entidad } \\
\text { federativa. }\end{array}$ & Población & INEGI \\
\hline CDES & $\begin{array}{l}\text { Flujo de crédito otorgado por la banca de } \\
\text { desarrollo por entidad federativa en } \\
\text { precios constantes. }\end{array}$ & $\begin{array}{l}\text { Millones de } \\
\text { pesos }\end{array}$ & Banxico \\
\hline CCOM & $\begin{array}{l}\text { Flujo de crédito otorgado por la banca } \\
\text { comercial por entidad federativa a precios } \\
\text { constantes. }\end{array}$ & $\begin{array}{l}\text { Millones de } \\
\text { pesos }\end{array}$ & Banxico \\
\hline TIIE & $\begin{array}{l}\text { Tasa de Interés Interbancaria de } \\
\text { Equilibrio a } 91 \text { días. }\end{array}$ & $\begin{array}{l}\text { Tasa } \\
\text { promedio } \\
\text { anual }\end{array}$ & INEGI \\
\hline TIBANC & $\begin{array}{l}\text { Tasa de interés bancaria neta en depósitos } \\
\text { a seis meses para personas físicas y } \\
\text { morales. }\end{array}$ & $\begin{array}{l}\text { Tasa } \\
\text { promedio } \\
\text { anual }\end{array}$ & INEGI \\
\hline LIQ & $\begin{array}{l}\text { Masa } \text { Monetaria }^{1} \text { (M1) a precios } \\
\text { constantes. }\end{array}$ & $\begin{array}{l}\text { Millones de } \\
\text { pesos }\end{array}$ & Banxico \\
\hline
\end{tabular}

Fuente: Elaboración propia.

Considerando el modelo propuesto, debe notarse que las últimas tres variables (TIIE, TIBANC y LIQ) están agregadas a nivel nacional, mientras el resto varían para cada una de las secciones cruzadas. Es decir, las tasas de interés y la masa monetaria son las mismas para todas las entidades federativas, solo varían en el tiempo. Esto implica que una de estas debe ser excluida de la ecuación, de lo contrario la estimación es imposible de llevar a cabo debido a problemas de colinealidad; en consecuencia, serán estimadas

\footnotetext{
${ }^{1}$ Además de un indicador de liquidez, la masa monetaria sirve como un instrumento para aproximar la inflación, debido a la estrecha relación que existe entre ambas variables. En el caso particular de este documento, debe considerarse que la inflación no es medida por entidad federativa en México.
} 
dos ecuaciones para poder observar los efectos de todas las variables. El método de estimación consiste en considerar que el elemento $c_{i}$ de la regresión está correlacionado arbitrariamente con alguna de las variables explicativas. Además, en las aplicaciones en donde las observaciones son unidades geográficas, como estados o provincias, no pueden tratarse como si fuera una muestra grande obtenida aleatoriamente, por lo que, la estimación debe llevarse a cabo con el supuesto de efectos fijos (Wooldridge, 2016). Esta precisión permite considerar una heterogeneidad inobservable entre las entidades federativas, lo cual hace posible obtener aproximaciones más precisas de los efectos ceteris paribus de cada uno de los coeficientes.

La estimación de los errores estándar de los coeficientes es robusta para heterocedasticidad y para correlación serial, a través de calcular los errores estándar para cada entidad federativa (cluster), lo cual permite corregir problemas de que los errores estén correlacionados en el tiempo, así como entre las secciones cruzadas.

\section{Resultados}

Los resultados de las estimaciones son mostrados en el cuadro 4, que contiene los coeficientes de las ocho ecuaciones que fueron estimadas. Por una parte, el PIB y el PIB per cápita son consistentes con estimaciones de otros trabajos, y en todos los casos tienen el signo esperado. El efecto del PIB es positivo para las empresas de todas las edades, lo que indica que el ingreso agregado o el tamaño del mercado es relevante para la existencia de firmas, no obstante, este coeficiente no es estadísticamente significativo para las empresas adultas. Por su parte, el PIB per cápita ejerce efectos que son consistentes con resultados obtenidos por Rangel y Torre (2015). Niveles altos de esta variable merman la cantidad de empresas en cualquiera de los estratos de edad, lo cual está relacionado con la elevación de los costos. Anteriormente, solo se había demostrado que el PIB afectaba a las empresas recién nacidas, sin embargo, las empresas jóvenes, adultas y mayores también se ven afectadas negativamente.

Cabe puntualizar que, dado que las variables están expresadas en logaritmos, los estimadores obtenidos representan la elasticidad de largo plazo de la variable dependiente con respecto a la independiente, lo que, desde el punto de vista del cálculo diferencial, es la pendiente. Acs, Desai y Hesse (2008) aseguran que la relación entre desarrollo y cantidad de empresas tiene una forma de "U"; es decir, en una primera etapa, a medida que la economía se desarrolla, la cantidad de empresas disminuye hasta alcanzar un punto mínimo, después de ese punto la cantidad de empresas aumenta conforme la economía se desarrolla. Considerando lo anterior, y que el PIB per cápita es utilizado como indicador de desarrollo, además que el coeficiente (la pendiente) es negativo, puede asegurarse que las entidades se hallan en el 
primer tramo de la curva en forma de "U", debido a que el aumento del PIB per cápita implica una disminución de la cantidad de empresas de todas las edades, en donde a las que menos afecta es a las adultas, mientras que las que más se ven afectadas son las jóvenes.

Es importante resaltar un aspecto importante con respecto al PIB y al PIB per cápita: el efecto del primero es mayor que el segundo para las empresas recién nacidas y jóvenes, mientras que para las empresas adultas y mayores sucede lo contrario. En otras palabras, el crecimiento económico provocará que existan más empresas recién nacidas y jóvenes que las que el desarrollo puede provocar que desaparezcan. Caso contrario para las adultas y mayores, en donde el desarrollo hará desaparecer mayor cantidad de empresas que las que el crecimiento económico puede generar.

La población desocupada propicia variaciones positivas en la cantidad de empresas de todas las edades. El grueso de la literatura se enfoca en considerar que el desempleo explica la formación de empresas, siendo estas una salida a dicha condición. Sin embargo, en el presente experimento esta variable no es concluyente, los coeficientes son considerablemente bajos y solo son significativos para las jóvenes y las mayores. Cabe señalar que esta variable está siendo utilizada rezagada, es decir, toma en cuenta los desocupados de un año anterior a la información de la cantidad de empresas, mientras que en el resto de los trabajos, se utiliza la información del mismo periodo para ambas variables.

En la literatura del emprendimiento, la relación entre desocupados y la creación de empresas ha sido ampliamente estudiada. Sin embargo, esta relación no limita el que la población que se halla en esta condición no pueda ocuparse en empresas de cualquier edad, de hecho, es un tema que no ha sido explorado hasta este momento. Lo que aquí se ha encontrado, es evidencia de que la población desocupada contribuye a que existan más empresas jóvenes y mayores, en un año posterior.

Las remesas son consideradas como una fuente de financiamiento informal, para este conjunto de datos, y ejercen un impacto negativo sobre la existencia de empresas de entre 5 y 10 años, con una significancia de 5\%.

La migración interestatal tiene un impacto positivo en las empresas recién nacidas. De acuerdo con Mungaray y Ramírez-Urquidy (2011), las personas que migran a un lugar determinado carecen de referencias profesionales y personales, así como de conexiones y contactos que les ayuden a encontrar un empleo en el mercado de trabajo; por lo tanto, están razonablemente motivados a emprender un negocio, lo cual corrobora que el coeficiente obtenido en el presente ejercicio, haya sido significativo solo para las empresas recién nacidas. 


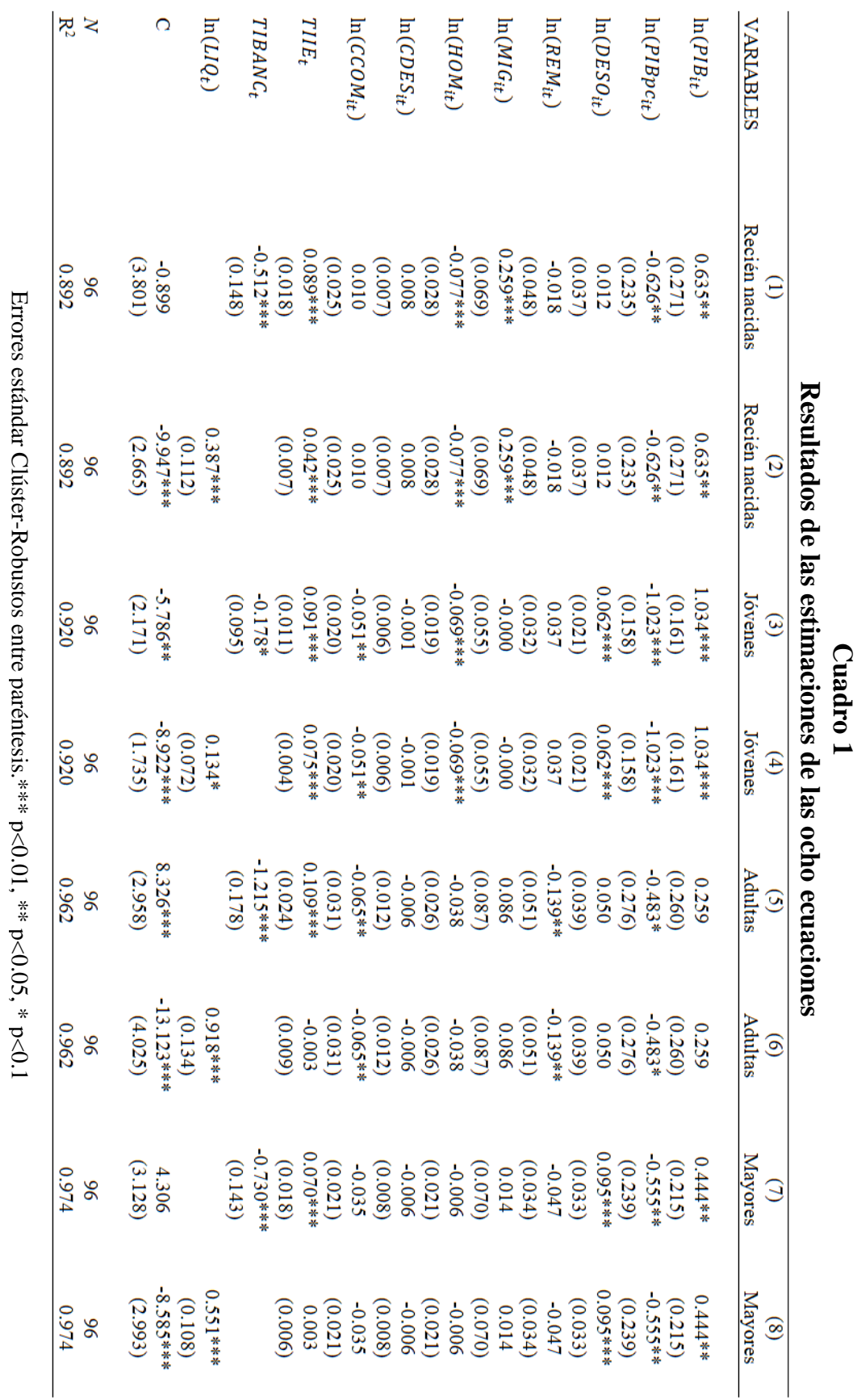


La inseguridad, medida a través de las defunciones por homicidio, ejerce un impacto negativo sobre las empresas recién nacidas y jóvenes. Esto sucede porque son empresas que se encuentran en una situación de mayor vulnerabilidad ante el crimen, que las empresas adultas o mayores, debido a que las firmas recién nacidas y jóvenes son de tamaño pequeño principalmente, lo cual provoca que estén más expuestas. Por su parte, las firmas adultas y mayores tienen capacidad superior de reacción ante situaciones de inseguridad, ya sea porque han generado experiencia a través de los años, o porque son de mayor tamaño, lo que es traducido en más recursos para hacer frente a este asunto.

La oferta de créditos de la banca de desarrollo no juega ningún papel significativo en la variación de empresas para ninguna edad. Si bien existen diferencias en los signos para las empresas nacidas y jóvenes con respecto a las adultas y mayores, ninguno de los resultados es concluyente, por lo que es necesario contar con más información para futuros trabajos, debido a que la banca de desarrollo en ocasiones es utilizada para el apalancamiento del crecimiento económico. No obstante, en México, una gran parte del crédito otorgado a través de las instituciones que pertenecen a este rubro, son destinados a vivienda y obra pública.

Por otro lado, la oferta del crédito de la banca comercial sí tiene un papel significativo en las empresas jóvenes y adultas, cuya cantidad de estas se ve afectada de manera negativa. La literatura sugiere que los emprendedores recurren a la banca comercial para financiar sus empresas, sin embargo, se desconoce cuál es la proporción de empresas recién nacidas cuyo capital proviene de un financiamiento formal o de uno informal. Por los resultados aquí obtenidos, puede considerarse que el primero es menos recurrente que el segundo, ya que existen estudios como el de Hernández-Trillo et al. (2005), y Rangel y Torre (2015) que indican que es menos accesible el financiamiento formal en México, por lo cual existen altos incentivos para recurrir a préstamos fuera del sistema financiero, a pesar de que eso implica arrancar en condiciones menos favorables para el éxito del negocio; la cuestión aquí es que, una mayor oferta de crédito de la banca comercial repercute en que existan menos empresas jóvenes y adultas.

La tasa de interés interbancaria tiene una relación positiva con la cantidad de empresas de cualquier edad; por cada punto porcentual que dicha tasa aumenta, se tiene un aumento que puede ser de $0.089 \%$ o $0.042 \%$ de empresas recién nacidas, depende de qué modelo se considere. ${ }^{2}$ Por su parte, el aumento en las empresas jóvenes es de $0.091 \%$ o $0.075 \%$, respectivamente. En el caso de las empresas adultas y mayores, este

\footnotetext{
${ }^{2}$ Debido a que la tasa de interés solo varía entre el tiempo y no entre las secciones cruzadas (entidades), esta cambia según la ecuación que se estime, ya sea cuando se considera junto con la tasa de interés bancaria o con la liquidez.
} 
coeficiente solo es significativo en una de las dos ecuaciones, puntualmente cuando es considerada la tasa de interés bancaria, en lugar de la liquidez. La cantidad de empresas adultas y mayores aumentará en $0.109 \%$ y $0.07 \%$, respectivamente, por cada aumento de un punto porcentual de la TIIE. Este resultado es contra intuitivo, debido a que suele suponerse que el aumento en la tasa de interés provocará un movimiento de dinero del mercado de bienes al mercado de capitales, sin embargo, este no es el caso, por el contrario, genera un efecto positivo. Este resultado está ligado con el que es obtenido mediante la variable de la tasa bancaria, la cual genera un efecto negativo en la existencia de firmas de cualquier edad. El impacto es considerablemente superior al que ejerce la TIIE sobre la cantidad de empresas; por ejemplo, para las empresas recién nacidas, un aumento de $1 \%$ en la tasa bancaria, provocará la disminución de $0.512 \%$ la cantidad de empresas recién nacidas; mientras que este efecto para las empresas jóvenes es de $0.178 \%$, para adultas de $1.215 \%$ y para las mayores de $0.73 \%$. El comportamiento de la cantidad de empresas con respecto a la tasa de interés bancaria es consistente con el hecho de que, si un emprendedor tiene la opción de invertir su dinero en un negocio o depositarlo en un banco para obtener algún rendimiento por este, y si el banco ofrece un pago superior a lo que el emprendedor espera obtener mediante un negocio, el emprendedor optará por mantener el dinero en el banco, debido a que el rendimiento está garantizado y el riesgo de perderlo es mínimo.

Finalmente, la liquidez de la economía o el dinero circulante ejerce un impacto positivo sobre la cantidad de empresas en cualquiera de las edades, aunque este resulta relativamente mayor para las empresas adultas y mayores. La masa monetaria es un reflejo de la política monetaria del país, que para el caso mexicano es conducido mediante la tasa de interés. Este resultado contrasta con el de esta última, ya que, si la liquidez tiene un impacto negativo sobre la existencia de empresas, la tasa de interés debería tener un efecto contrario, sin embargo, esto no sucede así. El comportamiento debiera ser similar al que sucede entre la tasa bancaria y la liquidez: ambos ejercen el efecto esperado sobre la existencia de empresas. La explicación de esto es que la TIIE sirve de referencia para la atracción o repulsión de capitales que no pertenecen al país, los cuales son intercambiados en cartera, en la que se puede invertir desde cualquier parte del mundo.

\section{Conclusiones}

En el presente trabajo, se puso a prueba la hipótesis de que los mismos factores afectan de manera diferente la existencia de empresas según su edad. Uno de los hallazgos más relevantes del presente documento es que, en el proceso de crecimiento y desarrollo que se encuentra la economía mexicana, se está generando que nazcan empresas y que las jóvenes se mantengan, ambas por encima de las que el propio proceso de desarrollo destruye como 
consecuencia de la elevación de los costos sobre la producción, así como los de la propia vida. De igual forma, el desarrollo de las entidades federativas genera que empresas adultas y mayores desaparezcan en mayor medida que las que el propio proceso de crecimiento económico puede incrementar. Este fenómeno es parte de un proceso que también ha sido observado en otros estudios, como el de Acs, et al. (2008).

Los dos factores que logran diferenciar más sus efectos sobre la cantidad de empresas, según su edad, son la migración y la inseguridad. Estos dos elementos solo afectan la existencia de empresas recién nacidas y jóvenes. La migración solo impacta la cantidad de empresas recién nacidas, por cada $1 \%$ que esta aumenta, la cantidad de empresas recién nacidas aumentará $0.259 \%$. Por su parte, un incremento de $1 \%$ de las defunciones por homicidio en las entidades federativas, provocará una disminución de $0.077 \%$ y $0.069 \%$ la cantidad de empresas recién nacidas y jóvenes, respectivamente. Este es un aspecto relevante para las entidades federativas, debido a que las estrategias de seguridad descansan en, buena medida, en las decisiones que son tomadas desde los gobiernos estatales; por lo tanto, una política indirecta que permita incrementar tanto la cantidad de empresas nacientes como la de empresas jóvenes (las que tienen entre 3 y 5 años), tiene que ver con la seguridad pública.

Uno de los factores que afecta en el mismo sentido la cantidad de empresas, independientemente de su edad, es la tasa de interés bancaria, lo cual es un aspecto a considerar desde la perspectiva de los diseñadores de política económica y de los organismos reguladores de la banca privada. Si bien, la tasa de interés está relacionada con aspectos de la seguridad de pago de los deudores, además de que en México cada institución financiera es libre de fijar la tasa de manera libre, muchas de estas decisiones están influenciadas por la política económica. En algunos casos, las instituciones financieras utilizan la TIIE como referencia, para entonces decidir la tasa que cobrarán; de modo que, por transitividad, los ajustes en la TIIE tendrán repercusiones en la tasa que fijan los bancos para otorgar créditos. A su vez, la TIIE está relacionada con la cantidad de dinero que circula en la economía, de tal manera que desde el banco central podrían tomarse decisiones, por una vía u otra de las aquí expuestas, con el objetivo de incrementar la cantidad de empresas en las entidades federativas.

Es importante considerar que el presente estudio es llevado a cabo a partir de datos agregados por entidad federativa, del cual pueden obtenerse elasticidades que permiten predecir aumentos o disminuciones de empresas en función de ciertos factores, como los aquí expuestos. No obstante, el presente análisis no debe considerarse como de supervivencia, ya que para ello son requeridos datos sobre la salida o cierre de empresas. Sin embargo, esto podrá considerarse como una posible extensión del presente trabajo. 
Otras posibles extensiones del presente trabajo pueden girar en torno a la composición espacial de la edad de las empresas, para determinar si, de alguna manera, la distribución sobre el territorio de estas y su edad sigue alguna distribución aleatoria, o el fenómeno sucede en forma de aglomeraciones. De igual manera, queda pendiente incorporar en el análisis una variable que controle los aspectos de innovación y capacidad emprendedora de las entidades federativas. Por último, se podrían considerar aspectos institucionales y de decisiones de política pública, lo que ayudaría - en el mismo sentido- a explicar con mayor alcance la formación o desaparición de empresas.

\section{Referencias}

[1] Acs, Z. J., Desai, S., y Hessels, J. (2008). Entrepreneurship, economic development and institutions. Small Business Economics(31), 219-234. doi:10.1007/s11187-008-9135-9

[2] Audretsch, D. B., y Acs, Z. J. (1994). New-Firm Startups, Technology, and Macroeconomic Fluctuation. Small Business Economics(6), 439-449.

[3] Bates, T. (1990). Entrepreneur Human Capital Inputs and Small Business Longevity. The Review of Economics and Statistics, LXXII(4), 551-559.

[4] Benavente, J. M., y Külzer, C. (2008). Creación y destrucción de empresas en Chile. Estudios de Economía, 35(2), 215-239.

[5] Bhattacharjee, A., Higson, C., Holly, S., y Kattuman, P. (2009). Macroeconomic Instability and Business Exit: Determinants of Failures and Acquisitions of UK Firms. Economica(76), 108-131. doi:10.1111/j.1468-0335.2007.00662.x

[6] Boeri, T., y Bellmann, L. (1995). Post-entry behaviour and the cycle: Evidence from Germany. International Journal of Industrial Organization, 13, 483-500.

[7] Dunne, P., y Hughes, A. (1994). Age, size, growth and survival: UK companies in the 1980s. The Journal of Industrial Economics, XLII(2), 115-140.

[8] Evans, D. S. (June de 1987). The relationship between firm growth, size and age; Estimate sfor 100 manufacturing industries. The Journal of Industrial Economics, $X X X V(4), 567-581$.

[9] Fort, T. C., Haltiwanger, J., Jarmin, R. S., y Miranda, J. (2013). How Firms Respond to Business Cycles: The Role of Firm Age and Firm Size. IMF Economic Review, 61(3), 520-559. doi:10.1057/imfer.2013.15

[10] Garcia-Quevedo, J., Pellegrino, G., y Vivarelli, M. (2014). RyD drivers and age: Are young firms different? Research Policy, 1-13. doi:http://dx.doi.org/10.1016/j.respol.2014.04.003

[11] Hernandez-Trillo, F., Pagan, J. A., y Paxton, J. (2005). Start-up Capital, Microenterprises and Technical Efficiency in Mexico. Review of Development Economics, 9(3), 434-447.

[12] Highfield, R., y Smiley, R. (1987). New business starts and economic activity. International Journal of Industrial Organization, 5, 51-66.

[13] Holtz-Eakin, D., Joulfaian, D., y Rosen, H. S. (Feb de 1994). Sticking it Out: Entrepreneurial Survival and Liquidity Constraints. The Journal of Political Economy, 102(1), 53-75.

[14] Huynh, K. P., y Petrunia, R. J. (2010). Age effects, leverage and firm growth. Journal of Economic Dynamics y Control(34), 1003-1013. doi:10.1016/j.jedc.2010.01.007 
[15] Ilmakunnas, P., y Topi, J. (1999). Microeconomic and Macroeconomic Influences on Entry and Exit of Firms. Review of Industrial Organization(15), 283-301.

[16] INEGI. (2014). Micro, pequeña, mediana y gran empresa: estratificación de los establecimientos. Instituto Nacional de Estadística, Geografía e Informática, Aguascalientes.

[17] Liu, J. (2004). Macroeconomic determinants of corporate failures: evidence from the UK. Applied Economics(36), 939-945. doi:10.1080/0003684042000233168

[18] Mansfield, E. (1962). Entry, Gibrat's Law, Innovation and The Growth of Firms. The American Economic Review, 52(5), 1023-1051.

[19] Mungaray, A., y Ramírez-Urquidy, M. (2011). Full and part-time entrepreneurship and the supply of entrepreneurial effort: Evidence from mexican microenterprises. Journal of Developmental Entrepreneurship, 16(4), 441-458. doi:10.1142/S1084946711001938

[20] Pfaffermayr, M., Stockl, M., y Winner, H. (2013). Capital Structure, Corporate Taxation and Firm Age. Fiscal Studies, 34(1), 109-135.

[21] Pfeiffer, F., y Reize, F. (2000). Business start-ups by the unemployed - an econometric analysis based on firm data. Labour Economics(7), 629-663.

[22] Rangel, E., y Torre, L. E. (2015). Determinants of the cost of starting a business in Mexico. North American Journal of Economics and Finance(34), 430-449.

[23] Sanchez-Vidal, J., y Martin-Ugedo, J. F. (2008). Edad y tamaño empresarial y ciclo de vida financiero. (S. Instituto Valenciano de Investigaciones Económicas, Ed.) Cartagena, Murcia, España.

[24] Tornhill, S., y Amit, R. (2003). Learning about Failure: Bankruptcy, Firm Age, and the Resource-Based View. Organization Science, 14(5), 497-509.

[25] Wooldridge, J. M. (2016). Introductory Econometrics: A modern approach (6th ed.). Boston, MA, USA: Cengage Learning. 